\title{
Complete Genome Sequence Resource for the Necrotrophic Plant-Pathogenic Bacterium Dickeya dianthicola 67-19 Isolated From New Guinea Impatiens
}

\author{
Yingyu Liu, ${ }^{1}$ Tyler C. Helmann, ${ }^{2}$ Paul Stodghill, ${ }^{1,2}$ and Melanie J. Filiatrault ${ }^{1,2, \dagger}$ \\ ${ }^{1}$ School of Integrative Plant Science, Section of Plant Pathology and Plant-Microbe Biology, Cornell \\ University, Ithaca, NY 14853 \\ ${ }^{2}$ Emerging Pests and Pathogens Research Unit, Robert W. Holley Center for Agriculture and Health, \\ Agricultural Research Service, United States Department of Agriculture, Ithaca, NY 14853
}

\begin{abstract}
New Guinea impatiens (NGI, Impatiens hawkeri) are popular bedding plants that can be affected by a number of pathogens. Using 16S rDNA sequencing and genus-specific PCR, we identified the first Dickeya dianthicola strain isolated from NGI presented with blackleg symptoms, herein designated as $D$. dianthicola 67-19. Here, we report a high-quality complete and annotated genome sequence of $D$. dianthicola $67-19$. The 4,851,809 bp genome was assembled with Nanopore reads and polished with Illumina reads, yielding $422 x$ and $105 \times$ coverage, respectively. This closed genome provides a resource for future research on comparative genomics and biology of $D$. dianthicola, which could translate to improved detection and disease management.
\end{abstract}

\section{Announcement}

Dickeya spp. are necrotrophic bacterial pathogens that can cause blackleg and soft rot disease on a wide range of host plants including field crops and ornamentals (Ma et al. 2007). Recent genomic analysis of Dickeya spp. isolated from Europe found little sequence diversity between $D$. dianthicola strains isolated from ornamentals and field crops, which suggests that $D$. dianthicola may have spread between greenhouse ornamentals and field crops (Toth et al. 2011). D. dianthicola was reported to cause blackleg disease of potatoes in Maine in 2015 (Jiang et al. 2016), and has since been reported in Morocco, Pakistan, Australia, and other parts of the United States (Boluk and Arif 2019; Nasaruddin et al. 2019; Oulghazi et al. 2017; Patel et al. 2019; Sarfraz et al. 2018; Wright et al. 2018). Here, we report the complete and annotated genome sequence of $D$. dianthicola $67-19$, which was originally isolated from $\mathrm{New}$ Guinea impatiens (Impatiens hawkeri) in Long Island, NY, U.S.A. (Liu et al. 2020).

D. dianthicola $67-19$ was cultured in Luria-Bertani (LB) medium at $28^{\circ} \mathrm{C}$ (per one liter of medium: $10 \mathrm{~g}$ of tryptone, $5 \mathrm{~g}$ of yeast extract, $10 \mathrm{~g} \mathrm{NaCl}$ ) (Bertani 1951). High molecular weight DNA was extracted from overnight bacterial cultures using a previously described method (Miller et al. 1988). DNA quantity was assessed using a NanoDrop 1000 spectrophotometer (Thermo Fisher Scientific, U.S.A.) and a Qubit 3.0 fluorometer (Thermo Fisher

${ }^{\dagger}$ Corresponding author: M. J. Filiatrault; melanie.filiatrault@usda.gov

The authors declare no conflict of interest. The funders had no role in the design of the study; in the collection, analyses, or interpretation of data, in the writing of the manuscript, or in the decision to publish the results. Mention of trade names or commercial products in this publication is solely for the purpose of providing specific information and does not imply recommendation or endorsement by the U.S. Department of Agriculture. USDA is an equal opportunity provider and employer.

Accepted for publication 14 October 2020.

This article is in the public domain and not copyrightable. It may be freely reprinted with customary crediting of the source. The American Phytopathological Society, 2021

\section{Funding}

This project was supported by the U.S. Department of Agriculture, Agricultural Research Service (USDA-ARS) project 8062-21000-042-00 and the USDA-ARS Floriculture and Nursery Research Initiative.

\section{Keywords}

Dickeya dianthicola, New Guinea impatiens, whole genome sequence, de novo assembly 
Table 1. Genome summary and average nucleotide comparisons of Dickeya dianthicola 67-19 to other Dickeya species type strains.

\begin{tabular}{lcccc} 
Dickeya strains & NCBI Accession & ANI (\%) & Genome size (bp) & Contig(s) \\
D. dianthicola 67-19 (this work) & CP051429 & NA & $4,851,809$ & 1 \\
D. dianthicola NCPPB 453 & GCA_000365305.1 & 97.43 & $4,676,060$ & 52 \\
D. solani IPO 2222 & GCA_001644705.1 & 92.40 & $4,919,833$ & 1 \\
D. dadantii NCPPB 898 & GCA_000406145.1 & 92.35 & $4,937,637$ & 52 \\
D. fangzhongdai DSM 101947 & GCA_002812485.1 & 92.10 & $5,032,447$ & 1 chromosome, 1 plasmid \\
D. undicola 2B12 & GCA_000784735.1 & 89.13 & $4,349,790$ & 77 \\
D. chrysanthemi NCPPB 516 & GCA_000406065.1 & 87.33 & $4,617,243$ & 34 \\
D. zeae NCPPB 2538 & GCA_000406165.1 & 85.38 & $4,563,790$ & 46 \\
D. aquatica 174/2 & GCA_900095885.1 & 82.23 & $4,501,560$ & 1 \\
D. paradisiaca NCPPB 2511 & GCA_000400505.1 & 81.70 & $4,631,867$ & 45 \\
\hline
\end{tabular}

a The average nucleotide identity (\%) between the $D$. dianthicola 67-19 strain sequenced in this study compared with other type strains of Dickeya species. Average nucleotide identity (ANI) was calculated using FastANI (Jain et al. 2018).

Scientific, U.S.A.). DNA integrity was assessed using the Agilent 2100 BioAnalyzer system (Agilent, U.S.A.).

Whole-genome sequencing technology capable of generating long reads (Nanopore) was used to obtain a complete scaffold of the genome and short reads (Illumina) were used to polish the assembled genome. All software were run with their default settings unless otherwise noted. For long-read sequencing, the DNA library was prepared using the Rapid Sequencing Kit SQK-RAD004 on a R9.4.1 SpotON flow cell with a MinION Mk1B device from Oxford Nanopore Technologies (United Kingdom). This generated 217,262 reads with a total length of 2,049,947,687 bp, and a genome coverage of 422x. The reads were basecalled using GUPPY v3.5.1 (Wick et al. 2019) and assembled using Flye v2.5 (Kolmogorov et al. 2019). For short-read sequencing, the DNA library was prepared using the NEBNext Ultra II FS DNA Library Prep Kit for Illumina with the NEBNext Multiplex Oligos (New England Biolabs, U.S.A.). AMPure XP beads (Beckman Coulter, U.S.A.) were used for DNA sizeselection and purification. The DNA library was sequenced on a MiSeq instrument (Illumina, U.S.A.) with the $2 \times 250$ bp mode, yielding 1,760,199 paired-end reads. Raw reads were processed to remove the adaptor and low-quality sequences using Trimmomatic v0.39 (reads were removed if quality value $[Q V] \leq 20$ or length $\leq 50$ ) (Bolger et al. 2014). Duplicate read pairs were removed using a customized Perl script (https://github.com/Sunhh/NGS_data_ processing/blob/master/drop_dup_both_end.pl) (Sun et al. 2017). BWA v0.7.17 (Li and Durbin 2009) and Pilon v1.22 (Walker et al. 2014) were used with default parameters to align the resulting $1,532,757$ high-quality trimmed Illumina read pairs to polish the draft genome for three rounds, with a genome coverage of 105x. Circlator v1.5.5 (Hunt et al. 2015) was used with the "fix start" argument to set the start of the genome at the $d n a A$ gene.

The final polished $D$. dianthicola $67-19$ genome is $4,851,809$ bp long with $55.93 \%$ GC content. It was annotated using the NCBI Prokaryotic Genome Annotation Pipeline (PGAP v4.11) (Tatusova et al. 2016). The resulting genome contains 4,352 genes in total, with 4,116 protein-coding genes, 132 pseudogenes, 8, 7, and 7 rRNA-coding sequences (5S, 16S, and $23 \mathrm{~S}$ rRNAs), 75 tRNAs, and 7 noncoding RNAs, and 1 clustered regularly interspaced short palindromic repeat (CRISPR) array. Whole-genome comparison of $D$. dianthicola 67-19 and other Dickeya type strains (Oulghazi et al. 2019; Parkinson et al. 2014) based on average nucleotide identity can be found in Table 1, including their genome sizes and number of contigs. Genome sequences were retrieved from publicly available sources (NCBI Resource Coordinators 2016). Average nucleotide identity (ANI) was calculated using FastANI (Jain et al. 2018). For prokaryotes, an ANI score of $95 \%$ or higher suggests that the two strains come from the same species (Konstantinidis et al. 2006). Based on ANI, D. dianthicola 67-19 showed the closest similarity to the $D$. dianthicola type strain NCPPB 453. This novel genome resource of $D$. dianthicola could aid in population studies of Dickeya, monitor future epidemiology, and assist the development of diagnostics tools for these destructive plant pathogens.

Data availability. The Dickeya dianthicola 67-19 genome sequence has been deposited at NCBI GenBank under the accession number CP051429. The Nanopore and Illumina raw reads have been deposited in the Sequence Read Archive (SRA) under the accession numbers SRR11531517 and SRR11531516, respectively. 


\section{Acknowledgments}

We thank Margery Daughtrey for providing helpful feedback on the manuscript. We thank the Genomics Facility of the Biotechnology Resource Center at the Cornell University's Institute of Biotechnology for their help with sequencing experiments.

\section{Literature Cited}

Bertani, G. 1951. Studies on lysogenesis. I. The mode of phage liberation by lysogenic Escherichia coli. J. Bacteriol. 62:293-300.

Bolger, A. M., Lohse, M., and Usadel, B. 2014. Trimmomatic: a flexible trimmer for Illumina sequence data. Bioinformatics 30:2114-2120.

Boluk, G., and Arif, M. 2019. First report of Dickeya dianthicola as a causal agent of bacterial soft rot of potato in Hawaii. Plant Dis. 103:2943.

Hunt, M., Silva, N. D., Otto, T. D., Parkhill, J., Keane, J. A., and Harris, S. R. 2015. Circlator: automated circularization of genome assemblies using long sequencing reads. Genome Biol. 16:294.

Jain, C., Rodriguez-R, L. M., Phillippy, A. M., Konstantinidis, K. T., and Aluru, S. 2018. High throughput ANI analysis of $90 \mathrm{~K}$ prokaryotic genomes reveals clear species boundaries. Nat. Commun. 9:5114.

Jiang, H. H., Hao, J. J., Johnson, S. B., Brueggeman, R. S., and Secor, G. 2016. First report of Dickeya dianthicola causing blackleg and bacterial soft rot on potato in Maine. Plant Dis. 100:2320.

Kolmogorov, M., Yuan, J., Lin, Y., and Pevzner, P. A. 2019. Assembly of long, errorprone reads using repeat graphs. Nat. Biotechnol. 37:540-546.

Konstantinidis, K. T., Ramette, A., and Tiedje, J. M. 2006. The bacterial species definition in the genomic era. Philos. Trans. R. Soc. Lond. B Biol. Sci. 361: 1929-1940.

Li, H., and Durbin, R. 2009. Fast and accurate short read alignment with Burrows-Wheeler transform. Bioinformatics 25:1754-1760.

Liu, Y., Vasiu, S., Daughtrey, M., and Filiatrault, M. 2020. First report of Dickeya diathicola causing blackleg on New Guinea Impatiens (Impatiens hawkeri) in New York State, USA. Plant Dis. https://doi.org/10.1094/PDIS-09-20-2020PDN

Ma, B., Hibbing, M. E., Kim, H.-S., Reedy, R. M., Yedidia, I., Breuer, J., Breuer, J., Glasner, J. D., Perna, N. T., Kelman, A., and Charkowski, A. O. 2007. Host range and molecular phylogenies of the soft rot enterobacterial genera Pectobacterium and Dickeya. Phytopathology 97:1150-1163.

Miller, S. A., Dykes, D. D., and Polesky, H. F. 1988. A simple salting out procedure for extracting DNA from human nucleated cells. Nucleic Acids Res. 16:1215.

Nasaruddin, A. S., Charkowski, A. O., Babler, B. N., Perna, N. T., and Glasner, J. D. 2019. First report of Dickeya dianthicola causing blackleg on potato in Texas. Plant Dis. 103:2121.
NCBI Resource Coordinators. 2016. Database resources of the National Center for Biotechnology Information. Nucleic Acids Res. 44(D1):D7-D19.

Oulghazi, S., Khayi, S., Lafkih, N., Massaoudi, Y., El Karkouri, A., El Hassouni, M., Faure, D., and Moumni, M. 2017. First report of Dickeya dianthicola causing blackleg disease on potato in Morocco. Plant Dis. 101:1671.

Oulghazi, S., Pédron, J., Cigna, J., Lau, Y. Y., Moumni, M., Van Gijsegem, F., Chan, K.-G., and Faure, D. 2019. Dickeya undicola sp. nov., a novel species for pectinolytic isolates from surface waters in Europe and Asia. Int. J. Syst. Evol. Microbiol. 69:2440-2444.

Parkinson, N., DeVos, P., Pirhonen, M., and Elphinstone, J. 2014. Dickeya aquatica sp. nov., isolated from waterways. Int. J. Syst. Evol. Microbiol. 64:2264-2266.

Patel, N., Baldwin, A. C., Patel, R. D., Kobayashi, D. Y., and Wyenandt, C. A. 2019. First report of Dickeya dianthicola causing blackleg and soft rot on potato (Solanum tuberosum) in New Jersey, U.S.A. Plant Dis. 103:146.

Sarfraz, S., Riaz, K., Oulghazi, S., Cigna, J., Alam, M. W., Dessaux, Y., and Faure, D. 2018. First report of Dickeya dianthicola causing blackleg disease on potato plants in Pakistan. Plant Dis. 102:2027.

Sun, H., Wu, S., Zhang, G., Jiao, C., Guo, S., Ren, Y., Zhang, J., Zhang, H., Gong, G., Jia, Z., Zhang, F., Tian, J., Lucas, W. J., Doyle, J. J., Li, H., Fei, Z., and Xu, Y. 2017. Karyotype stability and unbiased fractionation in the paleo-allotetraploid Cucurbita genomes. Mol. Plant 10:1293-1306.

Tatusova, T., DiCuccio, M., Badretdin, A., Chetvernin, V., Nawrocki, E. P., Zaslavsky, L., Lomsadze, A., Pruitt, K. D., Borodovsky, M., and Ostell, J. 2016. NCBI prokaryotic genome annotation pipeline. Nucleic Acids Res. 44:6614-6624.

Toth, I. K., van der Wolf, J. M., Saddler, G., Lojkowska, E., Hélias, V., Pirhonen, M., Tsror, L., and Elphinstone, J. G. 2011. Dickeya species: an emerging problem for potato production in Europe. Plant Pathol. 60:385-399.

Walker, B. J., Abeel, T., Shea, T., Priest, M., Abouelliel, A., Sakthikumar, S., Cuomo, C. A., Zeng, Q., Wortman, J., Young, S. K., and Earl, A. M. 2014. Pilon: an integrated tool for comprehensive microbial variant detection and genome assembly improvement. PLoS One 9:e112963.

Wick, R. R., Judd, L. M., and Holt, K. E. 2019. Performance of neural network basecalling tools for Oxford Nanopore sequencing. Genome Biol. 20:129.

Wright, D., Bwye, A., Banovic, M., Baulch, J., Wang, C., Hair, S., Hammond, N., Coutts, B., and Kehoe, M. 2018. First report of Dickeya dianthicola in potatoes in Australia. Plant Dis. 102:2029. 